Conflict of interest: KN receives grant support from NIAID, Food Allergy Research \& Education (FARE), End Allergies Together Allergenis, and Ukko Pharma; personal fees from Regeneron, AstraZeneca, ImmuneWorks, and Cour Pharmaceuticals; research sponsorsip from Novartis, Sanofi, Astellas, and Nestle; and research sponsorship for clinical trials from Genentech, Aimmune Therapeutics, DBV Technologies, AnaptysBio, Stallergenes-Greer, Regeneron, and Adare Pharmaceuticals. She is a data and Safety Monitoring Board member at Novartis and NHLBI; she cofounded Before Brands, Alladapt Immunotherapeutics, and ForTra; and she is a director for FARE and World Allergy Organization (WAO) Center of Excellence. SC receives grant support from CoFAR NIAID, Aimmune, DBV Technologies, Astellas, AnaptysBio, Novartis, Regeneron, StallergenesGreer, and Boehringer Ingelheim and is a scientific advisory board member for Alladapt Immunotherapeutics. $\mathrm{ML}$ is currently an employee at AnaptysBio. DP was an investigator for the study and received funds from AnaptysBio.

Copyright: (c) 2019, American Society for Clinical Investigation.

Submitted: July 3, 2019

Accepted: October 2, 2019

Published: November 14, 2019

Reference information: $/ C I$ Insight

2019;4(22):e131347.

https://doi.org/10.1172/jci.

insight.131347.

\section{Phase 2a randomized, placebo-controlled study of anti-IL-33 in peanut allergy}

\author{
Sharon Chinthrajah, ${ }^{1,2,3}$ Shu Cao, ${ }^{1,2}$ Cherie Liu, ${ }^{1,2}$ Shu-Chen Lyu, ${ }^{1,2}$ Sayantani B. Sindher, ${ }^{1,2,3}$ \\ Andrew Long, ${ }^{1,2}$ Vanitha Sampath, ${ }^{1,2}$ Daniel Petroni, ${ }^{4}$ Marco Londei, ${ }^{5}$ and Kari C. Nadeau ${ }^{1,2,3}$ \\ ${ }^{1}$ Sean N. Parker Center for Allergy and Asthma Research at Stanford University, ${ }^{2}$ Division of Pulmonary, Allergy and \\ Critical Care Medicine, and ${ }^{3}$ Division of Allergy, Immunology and Rheumatology, Stanford University, Stanford, California, \\ USA. ${ }^{4}$ ASTHMA Inc., Clinical Research Center, Northwest Asthma and Allergy Center, University of Washington, Seattle, \\ Washington, USA. ${ }^{5}$ AnaptysBio Inc., San Diego, California, USA
}

BACKGROUND. IL-33, found in high levels in participants with allergic disorders, is thought to mediate allergic reactions. Etokimab, an anti-IL-33 biologic, has previously demonstrated a good safety profile and favorable pharmacodynamic properties in many clinical studies.

METHODS. In this 6-week placebo-controlled phase 2a study, we evaluated the safety and the ability of a single dose of etokimab to desensitize peanut-allergic adults. Participants received either etokimab $(n=15)$ or blinded placebo $(n=5)$. Clinical tests included oral food challenges and skin prick tests at days 15 and 45 . Blood samples were collected for IgE levels and measurement of ex vivo peanut-stimulated T cell cytokine production.

RESULTS. Efficacy measurements for active vs. placebo participants at the day 15 and 45 food challenge (tolerating a cumulative $\mathbf{2 7 5}$ mg of peanut protein, which was the food challenge outcome defined in this paper) demonstrated, respectively, $73 \%$ vs. $0 \%(P=0.008)$ to $57 \%$ vs. $0 \%$ (ns). The etokimab group had fewer adverse events compared with placebo. IL-4, IL-5, IL-9, IL-13, and ST2 levels in CD4 ${ }^{+} \mathrm{T}$ cells were reduced in the active vs. placebo arm upon peanut-induced $\mathrm{T}$ cell activation ( $P=0.036$ for IL-13 and IL-9 at day 15$)$, and peanut-specific IgE was reduced in active vs. placebo $(P=0.014$ at day 15$)$.

CONCLUSION. The phase $2 \mathrm{a}$ results suggest etokimab is safe and well tolerated and that a single dose of etokimab could have the potential to desensitize peanut-allergic participants and possibly reduce atopy-related adverse events.

TRIAL REGISTRATION. ClinicalTrials.gov NCT02920021.

FUNDING. This work was supported by NIH grant R01AI140134, AnaptysBio, the Hartman Vaccine Fund, and the Sean N. Parker Center for Allergy and Asthma Research at Stanford University.

\section{Introduction}

Healthy immune tolerance to foods is an active process; a breakdown of this process is associated with sensitization to common foods as seen with food allergy, which are now estimated to affect approximately $8 \%$ of children (1) and $11 \%$ of adults (2) in the United States. Among adults, $45 \%$ are estimated to have multiple food allergies (2). Foods generally responsible for food allergy include milk, eggs, peanuts, soybeans, tree nuts, wheat, fish, and crustaceans (3). Peanut allergy, estimated to affect $1 \%-3 \%$ of the population, is associated with increased risk of severe anaphylactic reactions $(4,5)$ There are currently no approved treatments, and management of peanut allergy involves eliminating peanuts from the diet and treating reactions due to accidental exposures with antihistamines and epinephrine. Avoidance of allergens is difficult, and approximately $40 \%$ of patients with food allergies present with reactions due to accidental exposures each year (6). Anaphylaxis is among the leading causes for emergency department visits in the United States (6). Symptoms of exposure range from mild to severe and may include anaphylaxis, a severe and potentially life-threatening allergic reaction that requires immediate treatment. 


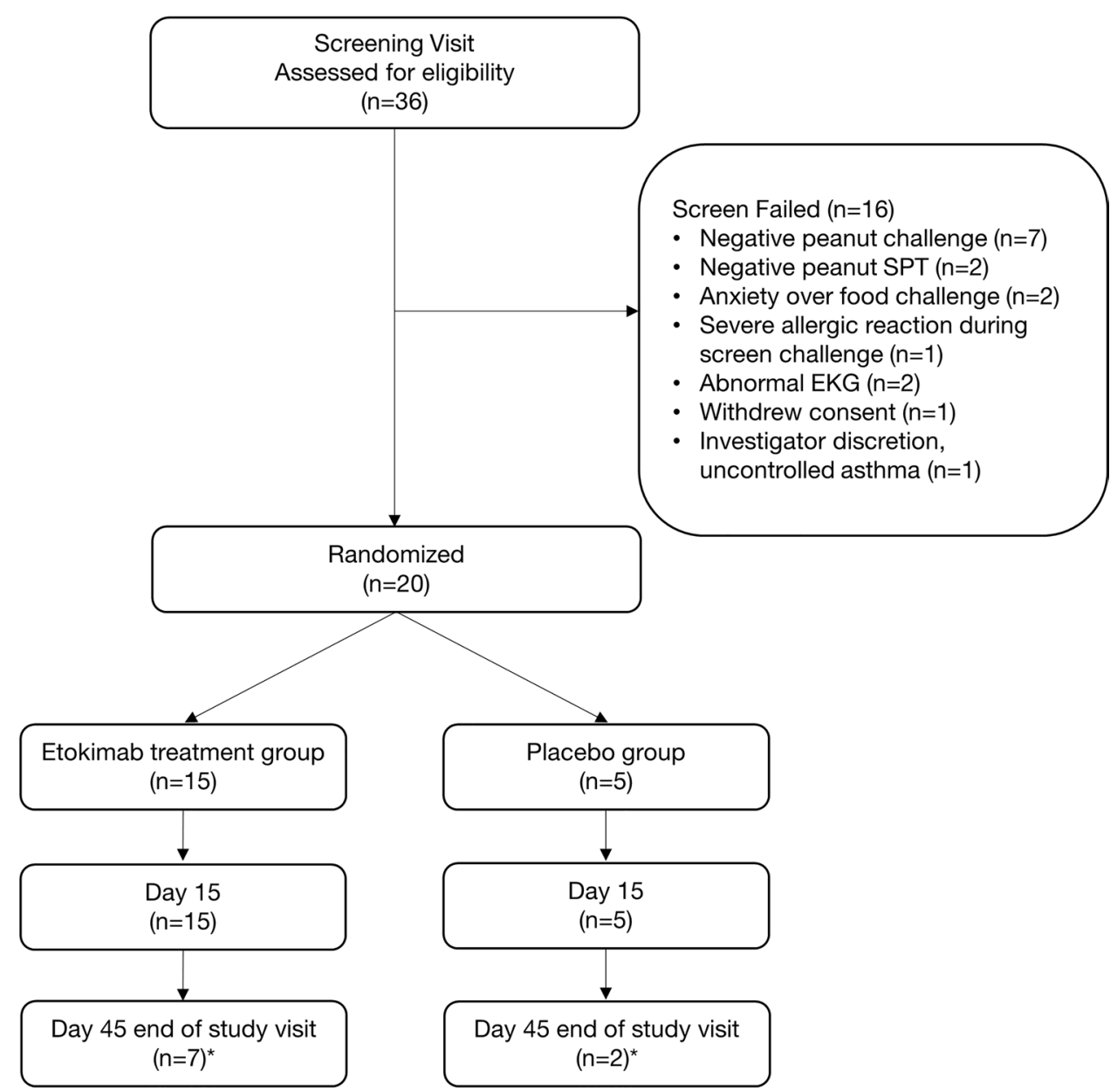

Figure 1. Participant enrollment consort diagram. EKG, electrocardiogram; SPT; skin prick tests. Asterisk indicates completed OFC.

Sensitization, one of the first steps in the development of food allergy, can occur not only through oral food exposure, but also via the skin. Food allergens first encounter barrier surfaces on the skin, gastrointestinal tract, or respiratory tract. Defects in skin barrier surfaces and a loss of integrity of these surfaces increase allergen permeability, leading to the production of proinflammatory epithelial-derived cytokines, such as IL-25, IL-33, and thymic stromal lymphopoietin (TSLP). Collectively, these cytokines are called alarmins and initiate an allergic inflammatory cascade, leading to allergic reactions in food allergy and other atopic diseases. Alarmins cause activation of type 2 innate lymphoid cells (ILC2s); differentiation of naive CD4 ${ }^{+} \mathrm{T}$ cells to Th2 cells; production of proinflammatory IL-4, IL-5, IL-9, and IL-13 cytokines; B cell isotype class switching from IgG to IgE; binding of IgE to FceRI receptors on mast cells or basophils with subsequent degranulation; and release of histamine and other proinflammatory allergic mediators $(7,8)$.

Biologics targeting alarmins, the Th2 cytokines (IL-4, IL-5, IL-9, IL-13), and IgE are in various stages of clinical development or have been approved for a number of atopic diseases. For example, the anti-IgE antibody omalizumab (Xolair), the anti-IL-5 monoclonal antibodies mepolizumab (Nucala) and reslizum$\mathrm{ab}$ (Cinqair), and the anti-IL-5R benralizumab (Fasenra) have been approved for allergic or eosinophilic asthma. Dupilumab (Dupixent), an antibody targeting IL-4R $\alpha$ (blocking IL-4 and IL-13 antibodies), has been approved for moderate-to-severe atopic dermatitis, as well as eosinophilic asthma. In clinical trials, adjuvant use of Xolair with oral immunotherapy has been shown to decrease time to maintenance dose in participants with food allergy (9). Since alarmins act upstream of Th2-associated cytokines and IgE production and have the potential to suppress multiple components of the Th2 response for food allergy and other atopic diseases, there is much interest in developing antibodies that target these molecules as therapeutic targets for food allergy and other atopic diseases (10-14). 
Screening (baseline) visit (7-14 days prior to Day 1$)(n=36)$

OFC, peanut and histamine skin prick test, ex vivo whole blood for peanut antigen challenge, biomarker analysis, and WBC.

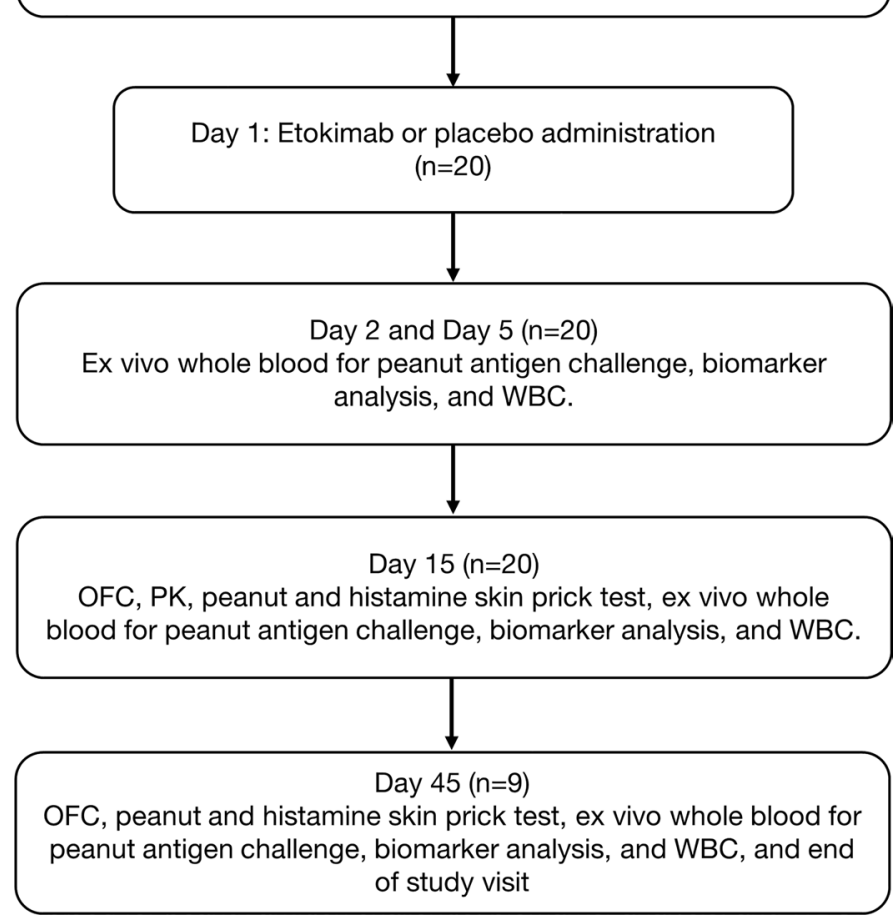

Figure 2. Study Design. OFC, oral food challenges; PK, pharmacokinetics; WBC, white blood cell count.

IL-33 is a member of the IL-1 cytokine family and a ligand for the orphan IL-1 family receptor, suppression of tumorigenicity 2 (ST2), a membrane-bound receptor, which instigates the production of different types of cytokines and chemokines that have crucial roles in the exacerbation of allergic diseases and inflammation. In addition to the membrane form of ST2, soluble ST2 (sST2) is produced by alternative splicing and acts as a decoy receptor to suppress IL-33-induced immune response (15). Induction of IL-33 can occur through a variety of mechanisms. For example, trefoil factor 2 (TFF2), an epithelial cell-derived repair molecule, was shown to be needed for the rapid production of IL-33 by lung epithelia, alveolar macrophages, and inflammatory DCs in infected mice (16). Activation of ST2 signaling by IL-33 triggers pleiotropic immune functions in multiple ST2-expressing immune cells, including macrophages, B cells, neutrophils, eosinophils, basophils, mast cells, Th2 cells, Tregs, and group 2 ILCs.

Table 1. Patient demographics

\begin{tabular}{lcc}
\hline Characteristics & $\begin{array}{c}\text { Active arm } \\
(\boldsymbol{n}=\mathbf{1 5})\end{array}$ & $\begin{array}{c}\text { Placebo arm } \\
(\boldsymbol{n}=\mathbf{5})\end{array}$ \\
$\begin{array}{l}\text { Sex (\% female) } \\
\begin{array}{l}\text { Age (median and range) } \\
\text { Peanut-specific lgE (kU/L) (median and range) }\end{array}\end{array}$ & $\begin{array}{c}67 \% \\
27 \text { yrs (19-54 yrs) }\end{array}$ \\
$\begin{array}{l}\text { CTD on baseline food challenge } \\
\text { (objective reactions) } \\
\text { (median and range) }\end{array}$ & $39.5(12-100)$ & 22 yrs (18-50 yrs) \\
$\begin{array}{l}\text { Other atopic conditions (asthma, allergic } \\
\text { rhinitis, eczema) }\end{array}$ & $175 \mathrm{mg}(5-175 \mathrm{mg})$ & $25 \mathrm{mg}(5-175 \mathrm{mg})$ \\
\hline
\end{tabular}

CTD, cumulative tolerated dose 
Table 2. Treatment emergent adverse events up to day 45.

\begin{tabular}{|c|c|c|}
\hline Adverse events & $\begin{array}{l}\text { Active arm } \\
(n=15) \\
n(\%)\end{array}$ & $\begin{array}{c}\text { Placebo arm } \\
\begin{array}{c}(n=5) \\
n(\%)\end{array}\end{array}$ \\
\hline Abdominal pain & $3(20.0)$ & $1(20.0)$ \\
\hline Headache & $4(26.7)$ & 0 \\
\hline Dizziness & $3(20.0)$ & 0 \\
\hline Fatigue & $1(6.7)$ & $1(20.0)$ \\
\hline Influenza-like illness & $2(13.3)$ & 0 \\
\hline Upper respiratory tract infection & $1(6.7)$ & $1(20.0)$ \\
\hline Adverse drug reaction & 0 & $1(20.0)$ \\
\hline Angioedema & 0 & $1(20.0)$ \\
\hline Arthralgia & $1(6.7)$ & 0 \\
\hline Asthma & 0 & $1(20.0)$ \\
\hline Eczema & $1(6.7)$ & 0 \\
\hline Food allergy & 0 & $1(20.0)$ \\
\hline Nasal congestion & $1(6.7)$ & 0 \\
\hline Nausea & 0 & $1(20.0)$ \\
\hline Rhinitis allergic & 0 & $1(20.0)$ \\
\hline Urinary tract infection & 0 & $1(20.0)$ \\
\hline Urticaria & $1(6.7)$ & 0 \\
\hline Viral upper respiratory tract infection & $1(6.7)$ & 0 \\
\hline Vomiting & 0 & $1(20.0)$ \\
\hline
\end{tabular}

Evidence of the role of IL-33 in atopic diseases, such as allergic rhinitis, asthma, atopic dermatitis, and food allergy, has been accumulating $(12,17-19)$. Many patients with food allergy have other atopic disorders; therefore, it was important in this phase 2 a pilot study to survey adverse events (AEs) related to atopy like asthma, allergic rhinitis and atopic dermatitis.

Several animal studies have shown that IL-33 is a major contributor in type- 2 inflammation in acute asthma. In epicutaneously ovalbumin-sensitized mice that were later orally challenged with ovalbumin, IL-33 was found to be essential for inducing IgE-dependent anaphylaxis in the gut (20). IL-33 is expressed more abundantly in subjects with asthma than in healthy individuals (21-24). Many antiIL-33 pathway inhibitors are being tested in clinical studies for allergy and/or asthma (NCT02999711, NCT03112577, NCT01928368).

Anti-IL-33 treatments in a mouse model of allergic rhinitis significantly reduced nose-scratching events, ameliorated skin denudation, and decreased eosinophilic infiltration and levels of serum total and OVA-specific IgE on i.p. and intranasal ovalbumin challenge, indicating that anti-IL-33 is a potential treatment for atopic diseases (25). Expressions of IL-33 and ST2 were also found to be significantly elevated in the plasma or epithelium of patients with allergic rhinitis $(26,27)$. Further, in patients with allergic rhinitis who showed clinical improvement after treatment with pollen immunotherapy, IL-33 was significantly decreased (28). Similar decreases in IL-33 were observed in serum and nasal lavages in children treated with sublingual immunotherapy for house dust mite allergy (29).

In a mouse model of atopic dermatitis (induced by 2, 4-dinitrochlorobenzene), those treated with anti-mouse IL-33 antibody showed improved symptoms, decreased eosinophils and mast cells infiltration, and reduced serum IgE levels (30). In humans, increased levels of IL-33 are also seen in patients with atopic dermatitis (30). Skin lesion studies of individuals with atopic dermatitis and house dust mite sensitization demonstrated upregulation of mRNA and protein expression of IL-33 (31). Currently, phase 2 clinical trials of anti-IL-33 for atopic dermatitis (NCT03533751) are underway.

In vitro studies of etokimab, a humanized immunoglobulin subtype $\mathrm{G} 1 / \kappa$ monoclonal antibody, indicate that it has a high affinity to human IL-33 and inhibits IL-33 activity, as determined by etokimab-mediated inhibition of IL-33/IL-12-induced IFN- $\gamma$ release in whole blood $(32,33)$. A phase 1 trial of etokimab in healthy volunteers indicated a favorable safety profile and pharmacodynamic properties. The terminal half-life of etokimab was approximately 372 hours, with comparable values across all doses (10-750 mg) 
Table 3. Treatment emergent adverse events by severity up to day 45.

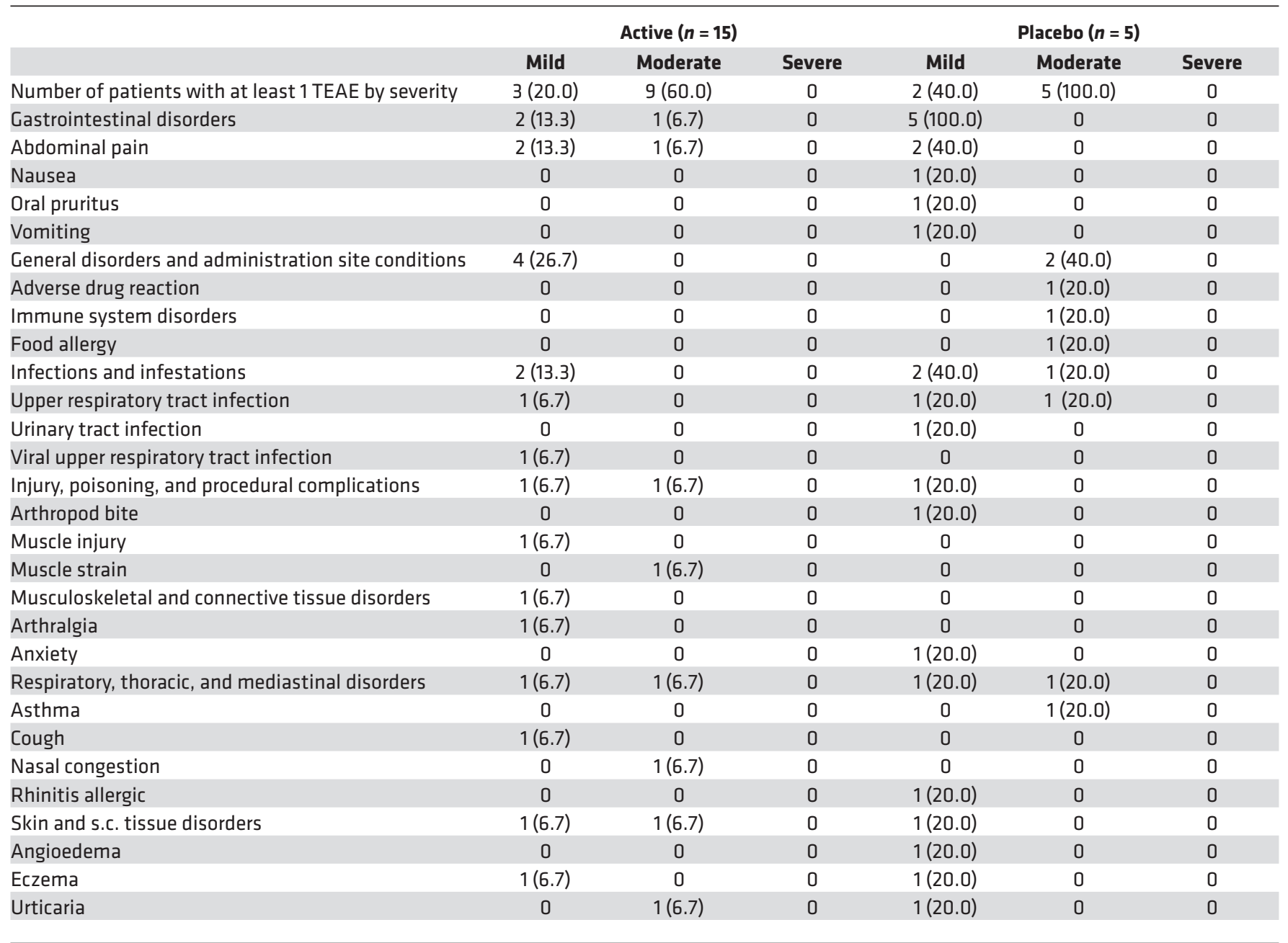

and regardless of route (i.v. or s.c.) of administration. No change in vital signs (blood pressure, heart rate, electrocardiogram, or body temperature) was noted. Hematology parameters, such as erythrocytes, WBC count, and platelet counts, were all within the normal range in participants dosed up to $750 \mathrm{mg}$. A single dose of $300 \mathrm{mg}$ administered i.v. was found to adequately suppress IL-33 function for 85 days after dosing, as measured by an ex vivo pharmacodynamic assay $(32,33)$.

Here, as a proof-of-concept phase 2 a study of etokimab in food allergy, we conducted a multicenter, randomized, double-blind, placebo-controlled phase 2 a clinical trial in adults with peanut allergy. The objectives of this placebo-controlled study were to determine the safety, tolerability, and change in standardized oral food challenge (OFC) following a single dose of etokimab vs. placebo in peanut-allergic adult participants. Achieving the primary endpoint was defined as an individual having no objective reaction to a cumulative tolerated dose (CTD) of $275 \mathrm{mg}$ of peanut protein using a standardized, validated, double-blind, placebo-controlled food challenge at day 15 (34). A follow-up phase of the study occurred between days 15 and 45 . Secondary endpoints included measurement of proinflammatory serum cytokines (IL-4, IL-5, IL-9, and IL-13), ST2, sST2, peanut- or histamine-specific skin prick test wheal size, and peanut and total IgE during the study.

\section{Results}

Patient characteristics. A total of 20 adult participants were randomized in a 3:1 ratio to receive either etokimab or placebo. Participant enrollment cohort diagram and the study design are presented in Figure 1 and Figure 2. All 20 patients completed the food challenge on day 15. The follow-up phase of the study 

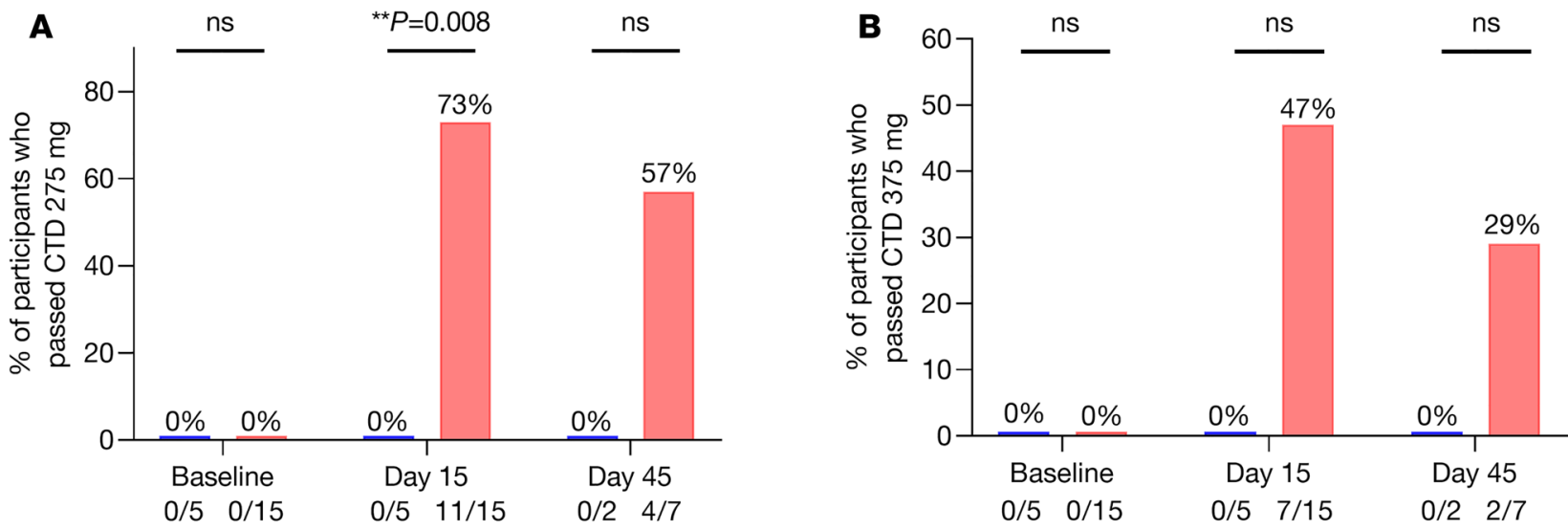

- Placebo Active

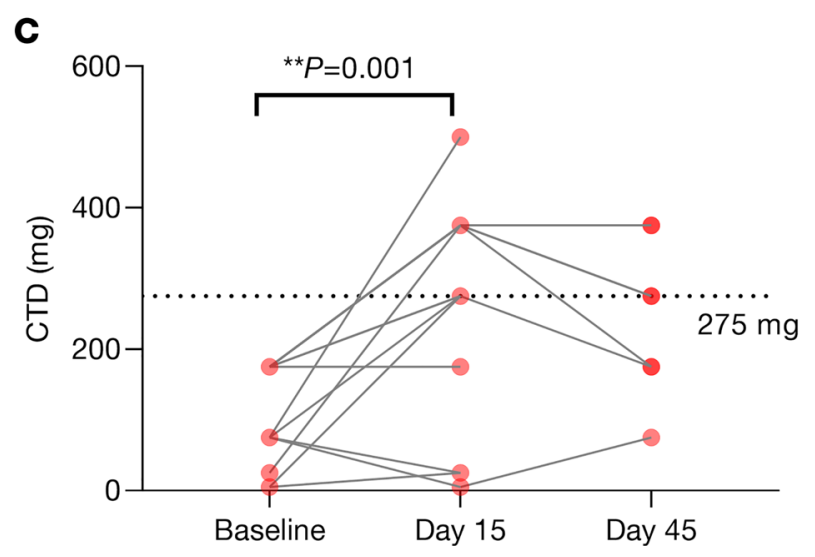

Figure 3. Oral food challenges. (A) Number of participants who passed the standardized OFC to cumulative 275 mg peanut protein. (B) Number of participants who passed the standardized OFC to cumulative $375 \mathrm{mg}$ peanut protein. (C) Participant-level spaghetti plot of CTD from baseline to day 45 for the active group. Fisher's exact test was used to compare the proportion of participants who passed food challenge between groups at each time point. The Wilcoxon matched-paired signed rank test was used to compare the CTD between baseline and day $15 .{ }^{*} P \leq 0.01$.

occurred after day 15. At day 45, 7 participants from the active treatment group and 2 from the placebo group returned to the research unit (a convenience sample based on ability to visit the clinic in their work schedules) and completed the food challenge on day 45. Participant demographics were summarized in Table 1 . The median age was 27 years for the active group and 22 years for the placebo group. The median CTD on the baseline food challenge were $175 \mathrm{mg}$ and $25 \mathrm{mg}$ for the active group $(n=15)$ and placebo $(n=$ 5) group, respectively. A total of $80 \%$ of participants from the active groups and $100 \%$ of participants from placebo group had at least a second atopic condition.

AEs. Treatment emergent AEs (TEAE) are summarized in Table 2 and Table 3 . The most frequent AE reported in etokimab-dosed participants was headache in 4 of 15 participants (26.7\%). In placebo-dosed participants, the most frequent AEs were atopy-related events (asthma, eczema, food allergy, and allergic rhinitis) in 3 of 5 participants (60\%). Atopy-related events were observed in only 1 of 15 participants $(7 \%)$ in the etokimab-dosed group. Compared with the placebo group, the participants in the active treatment arm experienced fewer moderate AEs (etokimab vs. placebo; $60 \%$ vs. $100 \%$, respectively). Etokimab was generally well tolerated during the study. No severe AEs (defined by predefined protocol and Common Terminology Criteria for Adverse Events [CTCAE]) were reported.

Food challenges. At baseline, all 20 participants (both the active and placebo group) met eligibility, which included reacting to standardized OFC, where a reaction was defined as an objective reaction to less than $275 \mathrm{mg}$ peanut protein. All standardized OFC results were reviewed by an independent, blinded expert reviewer. In the active group, 11 of $15(73 \%)$, and 4 of $7(57 \%)$ participants passed the OFC at day 15 and day 45 , respectively. Those who reached the $275 \mathrm{mg}$ threshold at day 45 had also reached this threshold at day 15 . None of the placebo participants passed the OFC at day 15 or at day 45 . 
A
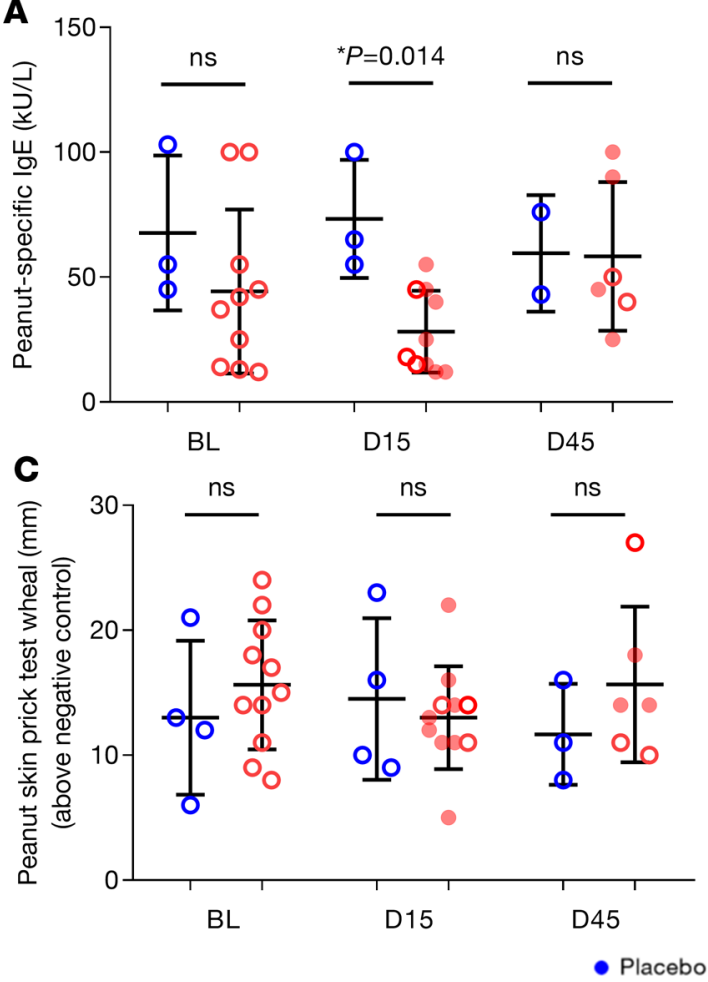
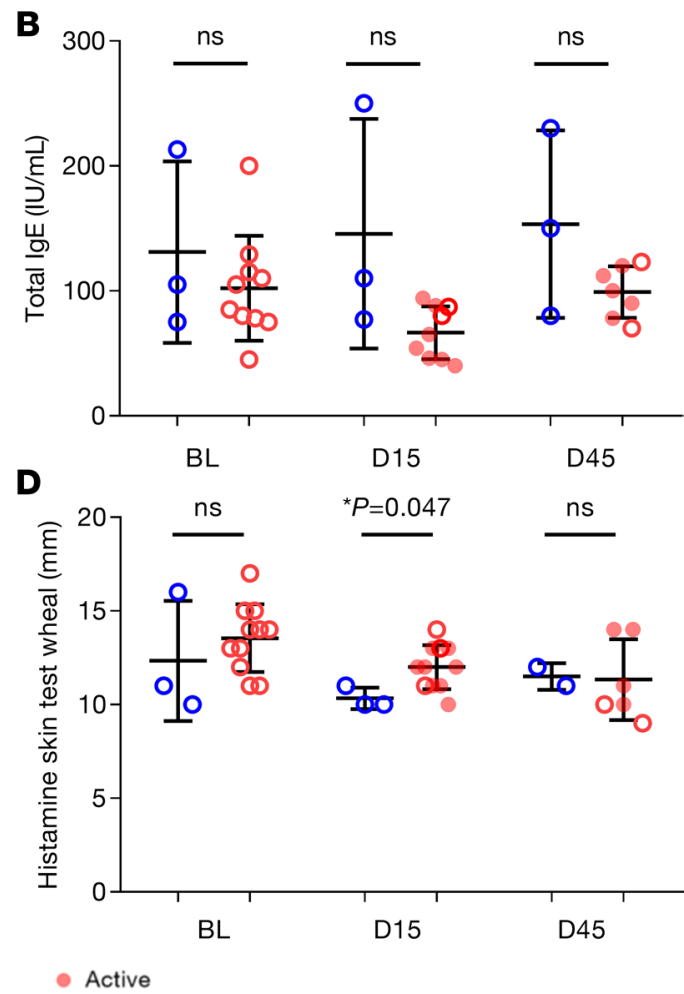

Figure 4. IgE and skin prick tests. Measurements of (A) peanut-specific IgE, (B) total IgE, (C) peanut skin prick test wheal sizes, and (D) histamine skin test wheal sizes for placebo (blue) and active (red) groups at each time point. Solid circles indicate subjects who passed food challenge on day 15 and day 45. All participants in the placebo group failed food challenge. The Mann-Whitney $U$ test was performed to compare the measurements between placebo and active groups at each time point. Mean $\pm \mathrm{SD}$ is presented. ${ }^{*} P \leq 0.05$.

However, since day 45 was part of the follow-up phase, only a few participants returned to try to complete the day 45 food challenge. Results indicate a significant increase in desensitization to peanut protein after a single i.v. administration of etokimab for the active group (Figure $3 \mathrm{~A} ; P=0.008$ ). We also compared the proportions of participants who passed the food challenge to a CTD of $375 \mathrm{mg}$. For participants in the active group, $47 \%$ on day 15 and $29 \%$ on day 45 passed the food challenge of cumulative $375 \mathrm{mg}$. Those who reached the $375 \mathrm{mg}$ threshold at day $45 \mathrm{had}$ also reached this threshold at day 15 . (Figure 3B). In addition, participants from the active group had a significant increase of median CTD on day 15 from baseline ( $275 \mathrm{mg}$ vs. $175 \mathrm{mg}, P=0.001$ ). There was no change for median CTD on day 45 compared with day 15 for active participants who underwent the food challenge on day 45 (275 $\mathrm{mg}$ vs. $275 \mathrm{mg}$ ) (Figure 3C). Furthermore, there was no significant change of median CTD from baseline (25 $\mathrm{mg})$ to day $15(75 \mathrm{mg})$ in the placebo group $(P=0.63)$.

Laboratory evaluations. The measurement of peanut-specific $\operatorname{IgE}$ and total $\operatorname{IgE}$ were shown to be lower in the active group compared with the placebo group over time; however, only the difference for peanut-specific IgE on day 15 reached statistical significance (baseline, $39.5 \mathrm{kU} / \mathrm{L}$ vs. $55 \mathrm{kU} / \mathrm{L}, P=$ 0.15 ; day $15,21.5 \mathrm{kU} / \mathrm{L}$ vs. $65 \mathrm{kU} / \mathrm{L}, P=0.014$; Figure 4 , A and B). In the active group, peanut-specific IgE level on day 15 was significantly lower than the measurement at baseline $(P=0.02)$. The peanut and histamine skin test wheal sizes among all participants were overall similar (Figure 4, C and D).

The measurements for IL- $4^{+}, \mathrm{IL}-5^{+}, \mathrm{IL}-9^{+}, \mathrm{IL}-13^{+}$, and $\mathrm{ST} 2^{+}$of total $\mathrm{CD} 4^{+} \mathrm{T}$ cells and of peanut-specific $\mathrm{CD}^{+} \mathrm{T}$ cells are presented in Figure 5 and Figure 6 . In general, treatment with etokimab decreased those intracellular cytokines tested. The active vs. placebo group showed lower percentages of IL- $4^{+}$cells of total $\mathrm{CD} 4^{+} \mathrm{T}$ cells on day $15(0.18 \%$ vs. $1.20 \%, P=0.036)$, and of peanut-specific $\mathrm{CD}^{+} \mathrm{T}$ cells on day $2(0.03 \%$ vs. $3.80 \%, P=0.006)$, day $5(0.14 \%$ vs. $4.10 \%, P=0.006)$, and day 45 $(2.50 \%$ vs. $4.30 \%, P=0.024)$ (Figure $5, \mathrm{~A}$ and $\mathrm{B})$. The percentages of IL- $5^{+}$cells of total $\mathrm{CD} 4^{+} \mathrm{T}$ cells were lower in the active vs. placebo group on day $5(0.09 \%$ vs. $0.42 \%, P=0.008)$ and day $15(0.13 \%$ vs. $0.40 \%, P=0.036$ ) (Figure 5, C and D). 

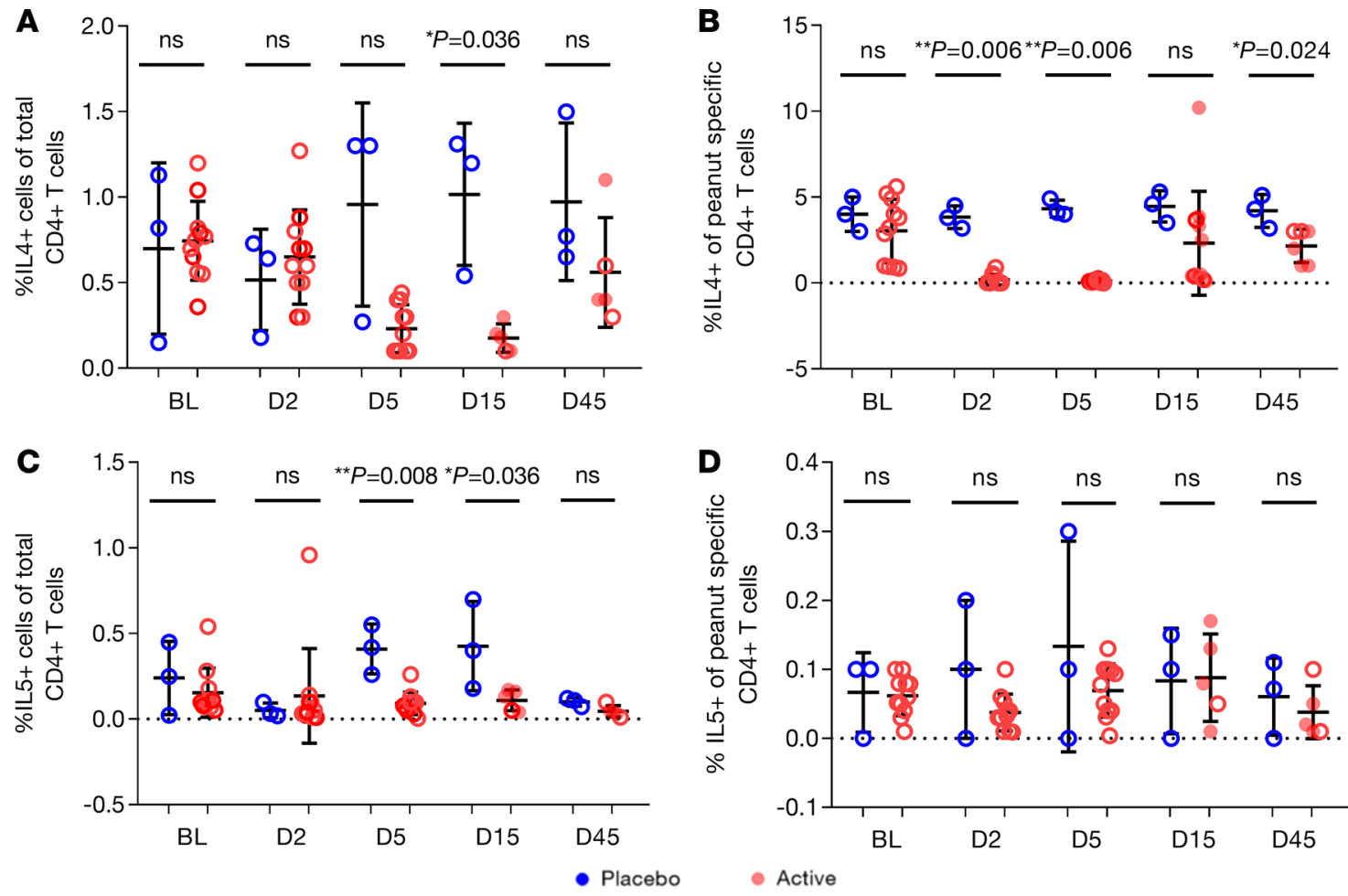

Figure 5. IL-4 and IL-5. Measurements of (A) IL- $4^{+}$cells of total CD4+ $T$ cells, (B) IL- $4^{+}$of peanut-specific CD4+ $\mathrm{T}$ cells, (C) IL-5 ${ }^{+}$cells of total CD4+ $\mathrm{T}$ cells, and (D) IL-5 of peanut-specific CD4+ $T$ cells. Solid circles indicate subjects who passed food challenge on day 15 and day 45 . All participants in the placebo group failed food challenge. The Mann-Whitney $U$ test was performed to compare the measurements between placebo and active groups at each time point. Mean $\pm \mathrm{SD}$ is presented. ${ }^{*} P \leq 0.05,{ }^{*} P \leq 0.01$.

For the measurements of IL-9+ $\mathrm{T}$ cells, the active vs. placebo group showed significantly lower percentages of IL- $9^{+}$cells of total CD $4^{+} \mathrm{T}$ cells on day $5(0.50 \%$ vs. $2.03 \%, P=0.006)$ and of peanut-specific $\mathrm{CD} 4^{+}$ T cells on day $2(0.26 \%$ vs. $2.08 \%, P=0.006)$, day $5(0.33 \%$ vs. $1.46 \%, P=0.006)$, day $15(0.32 \%$ vs. $3.00 \%$, $P=0.036)$, and day $45(0.40 \%$ vs. $3.50 \%, P=0.036)$ (Figure 6 , A and $\mathrm{B}$ ).

In addition, the active vs. placebo group showed significantly lower percentages of $\mathrm{IL}-13^{+}$cells of total CD $4^{+}$ T cells on day $15(0.02 \%$ vs. $0.30 \%, P=0.036)$ and of peanut-specific $\mathrm{CD} 4^{+} \mathrm{T}$ cells on day $2(2.32 \%$ vs. $10.00 \%$, $P=0.011)$, day $5(2.61 \%$ vs. $13.40 \%, P=0.006)$, day $15(2.49 \%$ vs. $13.00 \%, P=0.036)$, and day $45(3.40 \%$ vs. $11.00 \%, P=0.036$ ) (Figure $6, \mathrm{C}$ and $\mathrm{D}$ ). The active vs. placebo group also showed lower level of ST2 ${ }^{+}$cells of total $\mathrm{CD} 4^{+} \mathrm{T}$ cells on day $5(1.10 \%$ vs. $3.15 \%, P=0.011)$ and of peanut-specific $\mathrm{CD} 4^{+} \mathrm{T}$ cells on day $5(0.90 \%$ vs. $2.70 \%, P=0.003)$, day $15(0.45 \%$ vs. $2.30 \%, P=0.036)$, and day $45(1.50 \%$ vs. $3.00 \%, P=0.036)$ (Figure $6, \mathrm{E}$ and F). Percent total peanut-specific $\mathrm{CD} 4^{+} \mathrm{T}$ cells and ratio of $\mathrm{CD} 4^{+} \mathrm{T}$ cells $/ \mathrm{CD}^{+} \mathrm{T}$ cells did not change from baseline through days 45 (Figure 7). No change in sST2 was observed from baseline through day 15; there was also no statistical difference in sST2 expression between the placebo and active groups at any of the time points (Figure 8).

Individual, median, and mean plot of basophil/leukocyte counts were determined. No change in basophils was observed from baseline through day 15 . There were also no statistical differences between the placebo and active groups at any of the time points (Figure 9).

Pharmacokinetic data. We further investigated the association between the pharmacokinetic (PK) data, such as the maximum serum concentration $\left(\mathrm{C}_{\max }\right)$, and the time at which the $\mathrm{C}_{\max }$ was observed $\left(\mathrm{t}_{\max }\right)$, with cytokines levels and CTD on day 15. However, there was no significant association found between PK data and total IgE, percentage of IL- $13^{+}$cells of total CD4+ $\mathrm{T}$ cells, or CTD on day 15 (Figure 10, A-F).

\section{Discussion}

This placebo-controlled randomized phase 2a study is the first to our knowledge to use a monoclonal antibody targeting the alarmin IL-33 in peanut-allergic individuals. The results of this pilot study with a small group of participants $(n=20)$ are promising and show that a single dose of etokimab significantly improved desensitization in peanut-allergic participants (as determined by a standardized, validated food challenge in 
A
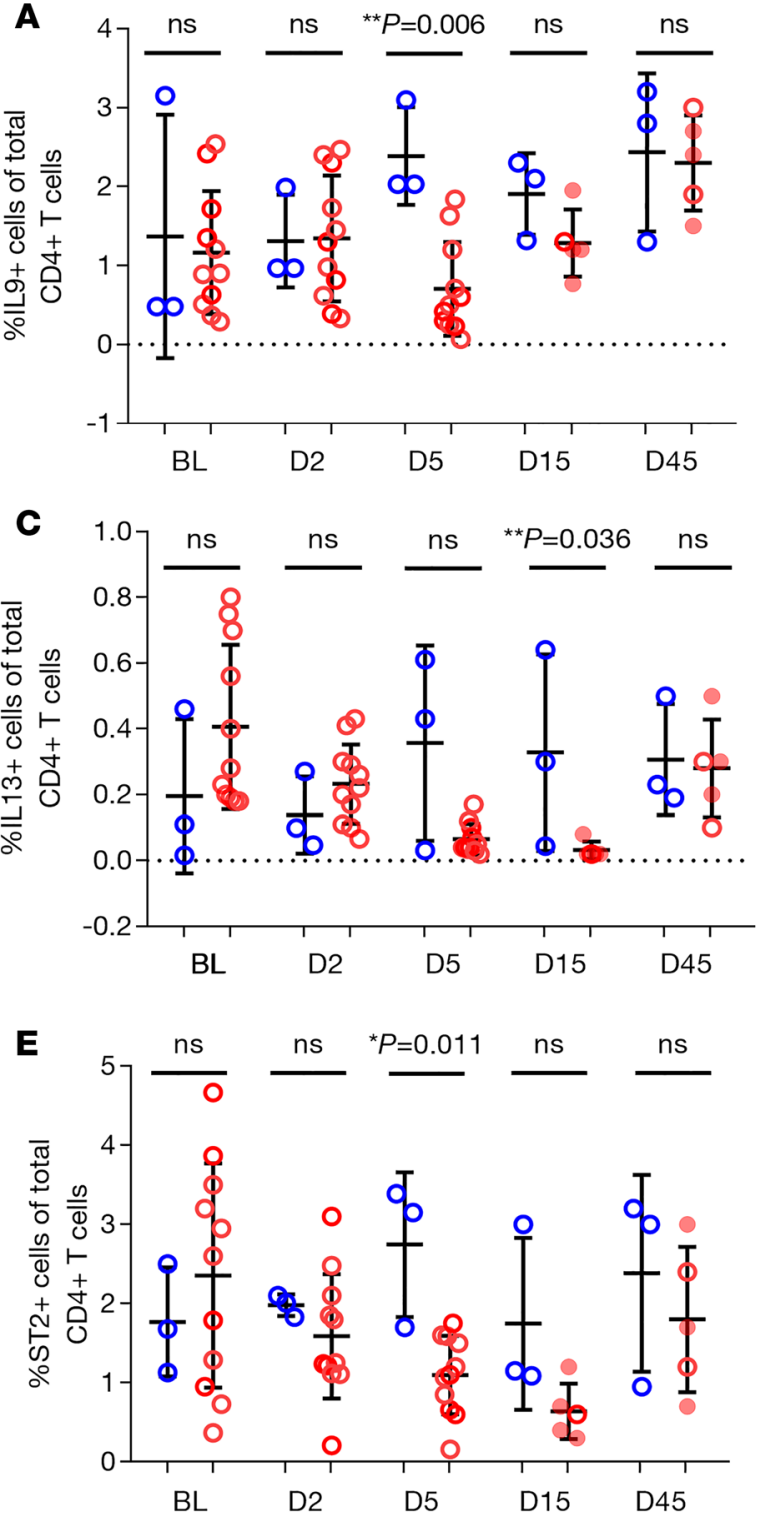

B
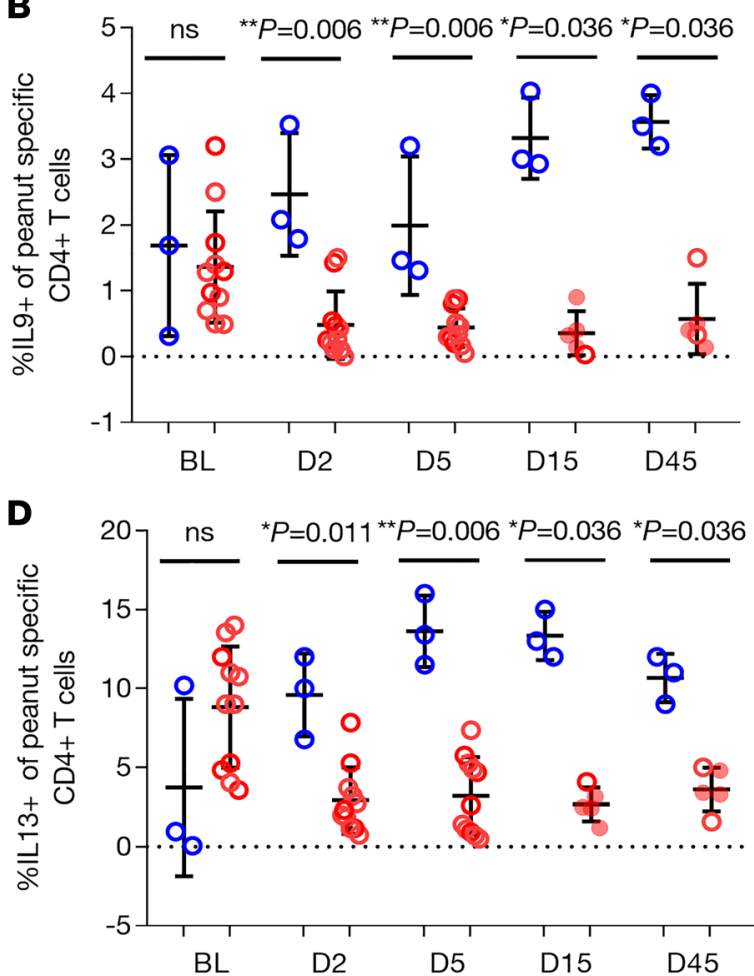

$\mathbf{F}$

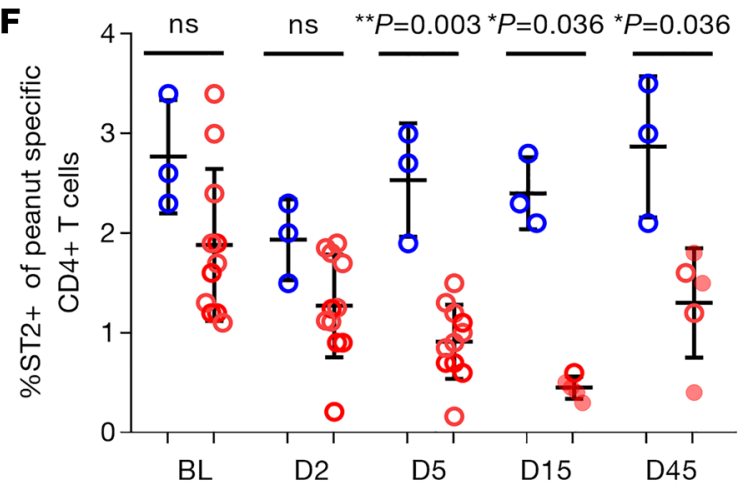

Figure 6. IL-9, IL-13, and ST2. Measurements of (A) IL-9+ cells of total CD4+ $4^{+}$cells, (B) IL-9+ of peanut-specific CD4+ $4^{+}$cells, (C) IL-13+ cells of total CD4+ ${ }^{+}$cells, (D) IL-13+ of peanut-specific CD4+ ${ }^{+}$cells, (E) ST2 ${ }^{+}$cells of CD4+ ${ }^{+}$cells, and (F) ST2 ${ }^{+}$of peanut-specific CD4+ ${ }^{+}$cells for placebo (blue) and active (red) groups at each time point. Solid circles indicate subjects who passed food challenge on day 15 and day 45 . All participants in the placebo group failed food challenge. The Mann-Whitney $U$ test was performed to compare the measurements between placebo and active groups at each time point. Mean $\pm \mathrm{SD}$ is presented. ${ }^{*} P \leq 0.05,{ }^{* *} P \leq 0.01$.

research facilities with independent review). There was also a trend in a reduction in atopy-related events in active vs. placebo groups during the study.

These results support previous evidence from animal and in vitro human studies that IL-33 blockade could be effective in inhibiting the allergic cascade. At the current time, there are no published studies of anti-IL-33 or anti-ST2 for food allergy in humans; however, studies for other atopic diseases such as atopic dermatitis (phase 2, NCT03533751), chronic rhinosinusitis with nasal polyps (phase 2, NCT03614923), and asthma (proof of concept, NCT03469934; phase 1, NCT02999711; phase 1, NCT03112577; phase 1, NCT01928368) are underway or have been completed.

In our study, we saw $73 \%$ and $57 \%$ increases in the tolerated threshold allergen dose of the active treatment group (day 15 and 45 , respectively), but we saw $0 \%$ in the placebo group on either of the 2 days. Biomarkers that assist with stratifying patients that respond well to anti-IL-33 antibody treatment will 

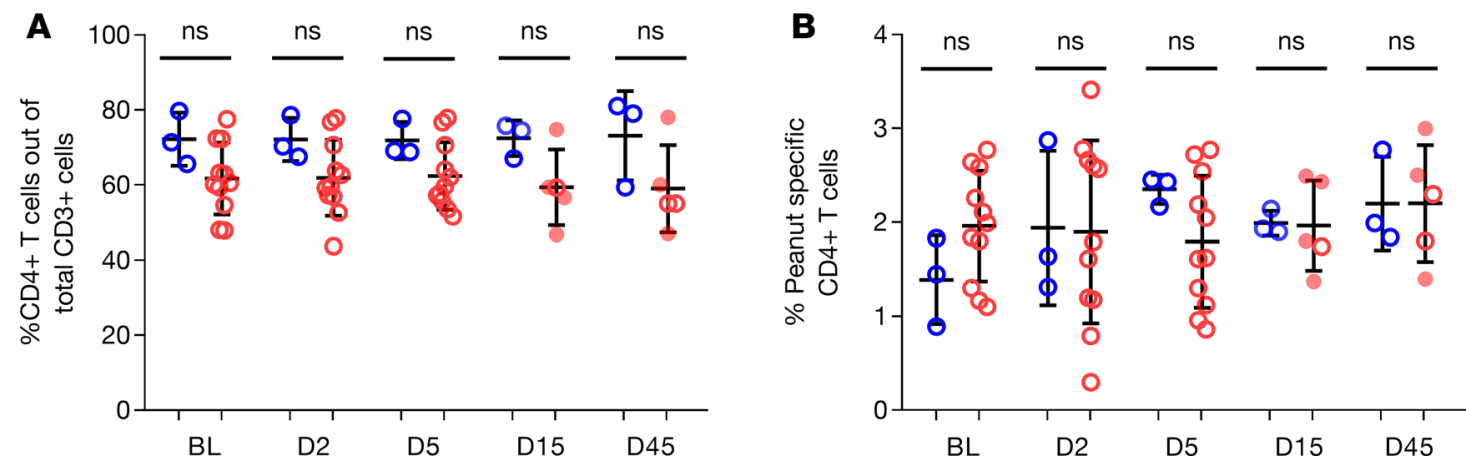

Figure 7. CD4+ $\mathbf{T}$ cells. Measurements of (A) CD4+ $T$ cell out of total $C D 3^{+}$cells and (B) peanut-specific CD4+ $T$ cells for placebo (blue) and active (red) groups at each time point. Solid circles indicate subjects who passed food challenge on day 15 and day 45 . All patients in the placebo group failed food challenge. The Mann-Whitney $U$ test was performed to compare the measurements between placebo and active groups at each time point. Mean \pm SD is presented.

assist with targeted therapy and precision medicine. It is interesting that self-reported atopy-related events (asthma, eczema, food allergy, and allergic rhinitis) were more common in the placebo group than the etokimab-treated group ( $60 \%$ vs. $7 \%$, respectively).

In our study, peanut-specific IgE was significant lower in the active group compared with the placebo group on day $15(P=0.014)$. In naive WT mice, administration of IL-33 amplifies IgE synthesis and triggers anaphylaxis in naive mice via IL-4 (35). Although we saw a trend toward a decrease in peanut-specific and total IgE with time in the active group, statistical significance was only observed at the day 15 time point from baseline for peanut-specific $\operatorname{IgE}(P=0.02)$. With immunotherapy, $\operatorname{IgE}$ decreases are seen typically after 12 months of treatment, so it was intriguing to see this level of change after 15 days (36). B cells have been found to express ST2, and perhaps IL-33 is needed, in part, for IgE synthesis (37). Since the half-life of $\operatorname{IgE}$ in the blood is 3 days, and since 15 days would represent 5 half-lives, there could be a significant decrease in the plasma, such as that seen in the small number of patients treated with anti-IL-33. We are currently pursuing mechanistic work to understand this decrease in IgE further.

In our study, etokinab use was associated with reduced levels of IL-4, IL-5, IL-9, and IL-13 in CD4 $4^{+} \mathrm{T}$ cells upon peanut stimulation. These results are encouraging and validate animal studies, which indicate that IL-33 increases Th2-type cytokines $(11,12,38)$. In vitro studies suggest that BM-derived mast cells activated with recombinant IL-33 produce cytokines IL-4 and IL-5 (39). When purified human naive or memory $\mathrm{CD}^{+} \mathrm{T}$ cells were stimulated with recombinant OX40L or TSLP-treated DCs in the presence of IL-33, increased production of proinflammatory cytokines IL-5, IL-9, and IL-13 were observed (40).

Therefore, we believe, based on these preliminary results, that the anti-IL-33 antibody, inhibiting IL-33 binding to ST2, could be working, in part, by decreasing those downstream allergic pathways dependent upon IL-33 (IL-5, IL-9, IL-13, IL-4) in CD4 ${ }^{+} \mathrm{T}$ cells. The effect on IgE is interesting and could be due to ST2 on B cells (37) and reduction in IgE synthesis through blockade of IL-33, although further studies are needed to test this hypothesis. It is interesting to note that there could be an ST2-independent mechanism in IL-33 modulation of B cell early development (41).

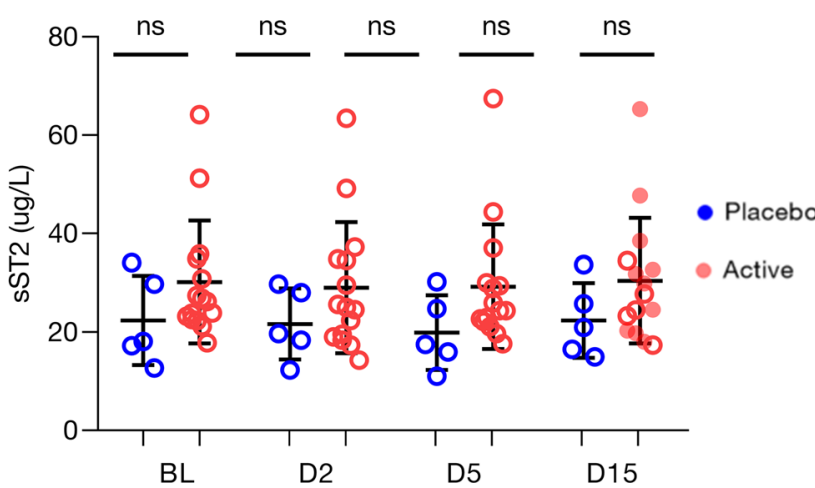

Figure 8. Individual, median, and mean plot of sST2. No change in SST2 was observed from baseline through day 15. There was also no statistical difference between the placebo and active groups at any of the time points. Solid circles indicate subjects who passed food challenge on day 15 and day 45. 


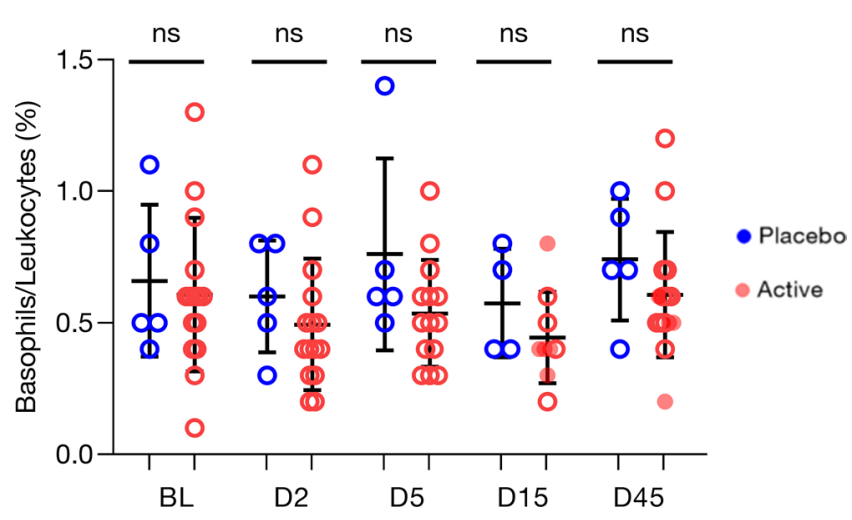

Figure 9. Individual, median, and mean plot of basophil/leukocyte counts. No change in basophils were observed from baseline through day 15 . There was also no statistical difference between the placebo and active groups at any of the time points. Mean and median are presented. Solid circles indicate subjects who passed food challenge on day 15 and day 45.

Our study had certain limitations. The sample size was small, which is typical of phase 2 trials in food allergy. Additionally, a few $(n=2)$ food challenges were open food challenges rather than double-blind placebo-controlled food challenges, and the challenges did not test for amounts greater than $375 \mathrm{mg}$ peanut protein. Other limitations included a short follow-up study period between days 15 and 45, limited sets of immune cells and their markers tested, and lack of basophil activation tests. Further, AE data were mostly based on participant self-reports, which can be highly variable.

Overall, the results of this phase 2 a study are promising. They suggest that etokimab is safe and well tolerated and that a single dose of etokimab could have the potential to desensitize peanut-allergic participants and possibly reduce atopy-related AEs. Larger and longer studies with multiple etokimab doses in food-allergic individuals are warranted.

\section{Methods}

Patient enrollment and study design. This study was a proof-of-concept, randomized, double-blind, placebo-controlled phase 2a clinical trial in adults with peanut allergy conducted at the Sean N. Parker Center for Allergy and Asthma Research at Stanford University and Seattle Children's Hospital between January, 2017 and April, 2018. Participants were evaluated for eligibility by OFCs in the screening visit at 7-14 days before treatment, which occurred on day 1 . Inclusion criteria included adult (older than 18 years) male or female participants (women of childbearing potential must be taking adequate contraceptive measures) with a clinical diagnosis of peanut allergy confirmed by OFCs. Exclusion criteria included a positive clinical reaction during the placebo OFC. On day 1, eligible participants were randomized at 3:1 to receive one i.v. dose of either etokimab $(300 \mathrm{mg} / 100 \mathrm{~mL})$ or placebo $(0.9 \%$ sodium chloride $[100 \mathrm{~mL}])$. OFCs were performed on days 1,14 , and 45 according to the PRACTALL consensus report (42). Escalating doses of peanut protein consisted of 5, 20, 50, 100, 100, 100, and $125 \mathrm{mg}$ (for a cumulative maximum dose of 500 mg). Symptoms were monitored using the OFC Symptom Scoring Assessment Tool. The total CTD of blinded peanut/placebo-tolerated and dosing step/threshold reached prior to reaction were recorded. Blood samples for measurement of ex vivo peanut-stimulated T cell cytokine intracellular production (IL-4, IL-5, IL-9, and IL-13) were obtained at baseline and at days 2, 5, 15, and 45. Peanut-specific T cells were identified as $\mathrm{CD} 154^{+} \mathrm{CD} 69^{+}$after peanut stimulation using standardized and published techniques. Antibodies against IL-5 (TRFK5) (BioLegend, catalog 504311) and IL-9 (MH9A3) (BD Biosciences, catalog 560807) were stained per manufacturer-recommended protocol. Further details of the methods used can be found in previous publications (43). Peanut-specific and total IgE, and peanut skin prick test and histamine skin tests, were performed at screening (baseline) and at days 15 and 45. Day 15 and day 45 blood samples and clinical tests (skin prick tests, OFCs) were permitted with a window period of 1 day. Safety and AEs were collected as per Good Clinical Practice guidelines. The consort diagram and study design schema are presented in Figure 1 and Figure 2. We have included data points for all participants for whom we had blood samples available.

Statistics. The primary endpoints were safety and tolerability, including AEs, serious AEs (SAEs), and severity of AEs (mild, moderate, and severe). The secondary endpoints were clinical laboratory 

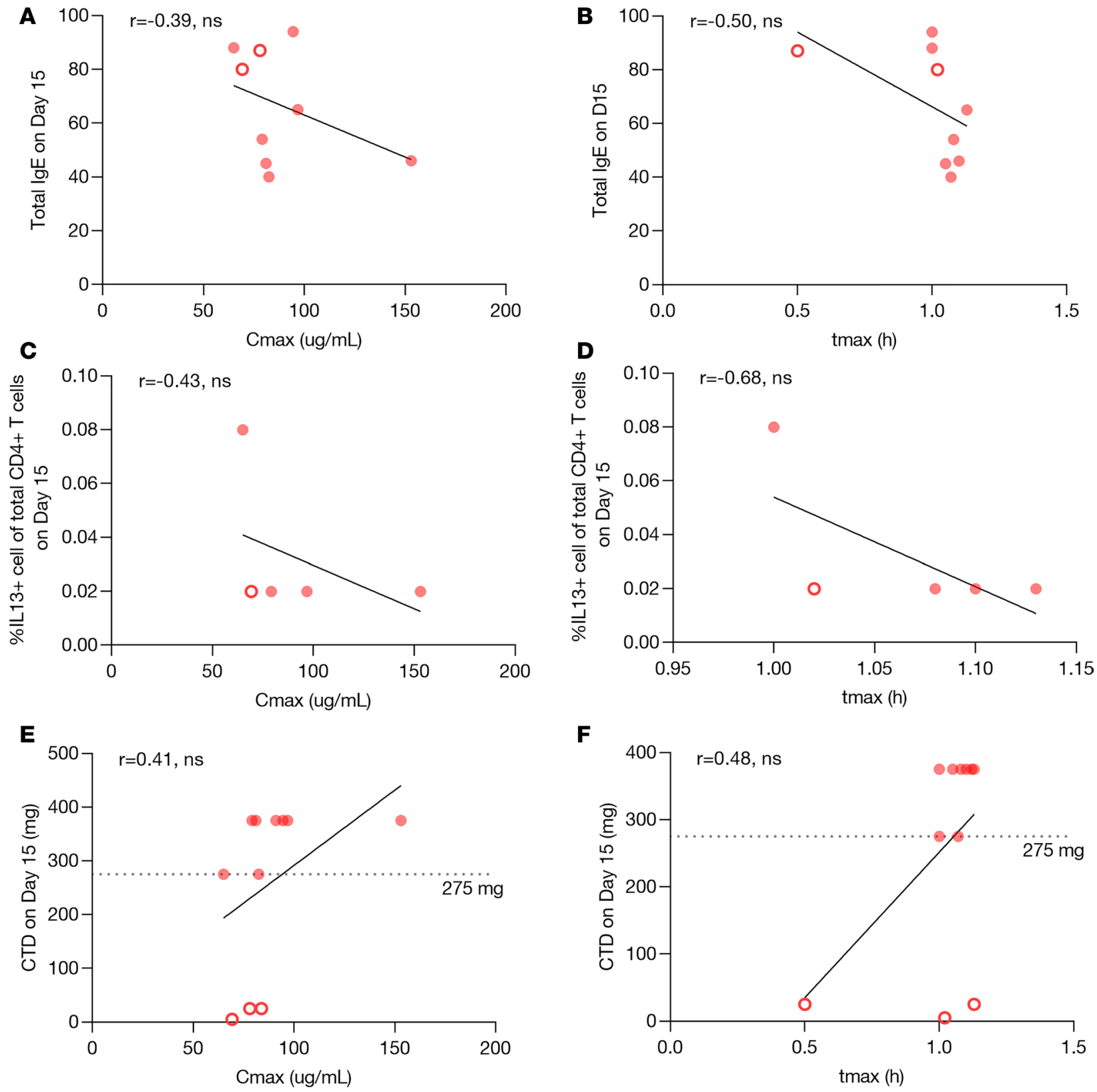

Figure 10. $\mathbf{C}_{\max }$ and $\mathbf{t}_{\max }$. Linear regression between (A) $C_{\max }$ and total IgE, (B) $t_{\max }$ and total IgE, (C) $C_{\max }$ and IL-13 level of total CD4 ${ }^{+} T$ cells, (D) $t_{\max }$ and IL-13 level of total CD4+ $T$ cells, (E) $C_{\max }$ and CTD, and (F) $t_{\max }$ and CTD. There was no significant association between the measurements and $C_{\max }$ or $t_{\max }$. Open circles indicate subjects who failed food challenge on day 15; solid circles indicate subjects who passed food challenge on day 15.

endpoints at specific time points. Number and percentage of AEs were presented for each treatment by preferred term and system organ class of the current Medical Dictionary for Regulatory Authorities (MedDRA). The OFC CTD were summarized and illustrated using a bar charts. Appropriate descriptive statistics were summarized for the observed values at each scheduled assessment and for the corresponding change from baseline. Baseline was defined as the last assessment before administration of the IP. The Mann-Whitney $U$ test was performed to compare the median laboratory measurements between the placebo and active groups at each time point. The Wilcoxon matched-paired signed rank test was used to compare the laboratory measurements or CTD values between different time points within treatment group. Linear regression was used to evaluate the association between PK data with cytokines levels and CTD on day 15. The analyses were conducted using GraphPad Prism version 8.0.2 for Windows. All tests were 2-sided at a significance level of $0.05 . P$ values were not adjusted for multiple testing, since this was a pilot study. 
Study approval. The trial protocol was approved by the IRB at the participating centers. The trial was registered at clinicaltrials.gov before initiating recruitment (NCT02920021). Written informed consent was received from participants prior to inclusion in the study.

\section{Author contributions}

S. Chinthrajah, KCN, and ML designed the study and wrote and critically reviewed the manuscript. S. Cao analyzed data. CL performed the study and collected data. S-CL conducted the laboratory work. VS wrote and critically reviewed the manuscript. SBS helped conduct the study and write the manuscript. AL and DP helped conduct the study.

\section{Acknowledgments}

This work was supported by NIH grant R01AI140134, AnaptysBio, the Hartman Vaccine Fund, and the Sean N. Parker Center for Allergy and Asthma Research at Stanford University. KN is a grant awardee for NIAID, NHLBI, NIEHS, and EPA.

Address correspondence to: Kari C. Nadeau, Sean N. Parker Center for Allergy and Asthma Research at Stanford University, 269 Campus Drive, CCSR 3215, MC 5366, Stanford, California 94305-5101, USA. Phone: 650.724.6780; Email: knadeau@stanford.edu.

1. Gupta RS, et al. The Public Health Impact of Parent-Reported Childhood Food Allergies in the United States. Pediatrics. 2018;142(6):e20181235.

2. Gupta RS, et al. Prevalence and Severity of Food Allergies Among US Adults. JAMA Netw Open. 2019;2(1):e185630.

3. Nwaru BI, et al. Prevalence of common food allergies in Europe: a systematic review and meta-analysis. Allergy. 2014;69(8):992-1007.

4. Gupta RS, et al. The prevalence, severity, and distribution of childhood food allergy in the United States. Pediatrics. 2011;128(1):e9-17.

5. Peters RL, et al. The prevalence of food allergy and other allergic diseases in early childhood in a population-based study: HealthNuts age 4-year follow-up. J Allergy Clin Immunol. 2017;140(1):145-153.e8.

6. Rudders SA, Banerji A, Vassallo MF, Clark S, Camargo CA. Trends in pediatric emergency department visits for food-induced anaphylaxis. J Allergy Clin Immunol. 2010;126(2):385-388.

7. Chinthrajah RS, Hernandez JD, Boyd SD, Galli SJ, Nadeau KC. Molecular and cellular mechanisms of food allergy and food tolerance. J Allergy Clin Immunol. 2016;137(4):984-997.

8. Sampath V, Sindher SB, Zhang W, Nadeau KC. New treatment directions in food allergy. Ann Allergy Asthma Immunol. 2018;120(3):254-262.

9. Andorf S, et al. Anti-IgE treatment with oral immunotherapy in multifood allergic participants: a double-blind, randomised, controlled trial. Lancet Gastroenterol Hepatol. 2018;3(2):85-94.

10. Roan F, Obata-Ninomiya K, Ziegler SF. Epithelial cell-derived cytokines: more than just signaling the alarm. J Clin Invest. 2019;129(4):1441-1451.

11. Leung DYM. Cutaneous allergy: control that itch-scratch cycle! Ann Allergy Asthma Immunol. 2019;123(2):115.

12. Mahlakõiv T, Artis D. Allergen Exposure: When Timing Is Everything. Immunity. 2016;45(6):1188-1190.

13. Leyva-Castillo JM, et al. Mechanical Skin Injury Promotes Food Anaphylaxis by Driving Intestinal Mast Cell Expansion. Immunity. 2019;50(5):1262-1275.e4.

14. de Kleer IM, et al. Perinatal Activation of the Interleukin-33 Pathway Promotes Type 2 Immunity in the Developing Lung. Immunity. 2016;45(6):1285-1298.

15. Pusceddu I, Dieplinger B, Mueller T. ST2 and the ST2/IL-33 signalling pathway-biochemistry and pathophysiology in animal models and humans. Clin Chim Acta. 2019;495:493-500.

16. Wills-Karp M, et al. Trefoil factor 2 rapidly induces interleukin 33 to promote type 2 immunity during allergic asthma and hookworm infection. J Exp Med. 2012;209(3):607-622.

17. Ding W, Zou GL, Zhang W, Lai XN, Chen HW, Xiong LX. Interleukin-33: Its Emerging Role in Allergic Diseases. Molecules. 2018;23(7):E1665

18. De Grove KC, et al. IL-33 signalling contributes to pollutant-induced allergic airway inflammation. Clin Exp Allergy. 2018;48(12):1665-1675.

19. Mathews JA, et al. IL-33 Drives Augmented Responses to Ozone in Obese Mice. Environ Health Perspect. 2017;125(2):246-253.

20. Muto T, et al. The role of basophils and proallergic cytokines, TSLP and IL-33, in cutaneously sensitized food allergy. Int Immunol. 2014;26(10):539-549.

21. Raeiszadeh Jahromi S, et al. Serum levels of IL-10, IL-17F and IL-33 in patients with asthma: a case-control study. J Asthma. 2014;51(10):1004-1013.

22. Momen T, Ahanchian H, Reisi M, Shamsdin SA, Shahsanai A, Keivanfar M. Comparison of Interleukin-33 Serum Levels in Asthmatic Patients with a Control Group and Relation with the Severity of the Disease. Int J Prev Med. 2017;8:65.

23. Poulsen NN, et al. Airway Interleukin-33 and type 2 cytokines in adult patients with acute asthma. Respir Med. 2018;140:50-56.

24. Al-Sajee D, et al. Expression of IL-33 and TSLP and Their Receptors in Asthmatic Airways after Inhaled Allergen Challenge. Am J Respir Crit Care Med. 2018;198(6):805-807. 
25. Kim YH, et al. Anti-IL-33 antibody has a therapeutic effect in a murine model of allergic rhinitis. Allergy. 2012;67(2):183-190.

26. Kamekura R, Kojima T, Takano K, Go M, Sawada N, Himi T. The role of IL-33 and its receptor ST2 in human nasal epithelium with allergic rhinitis. Clin Exp Allergy. 2012;42(2):218-228.

27. Qiao Y, Chen J. Serum levels of IL-31, IL-33 and ST2 in allergic rhinitis of children in China. Cell Mol Biol (Noisy-le-grand). 2018;64(12):52-55.

28. Nasr WF, Sorour SS, El Bahrawy AT, Boghdadi GS, El Shahaway AA. The Role of the Level of Interleukin-33 in the Therapeutic Outcomes of Immunotherapy in Patients with Allergic Rhinitis. Int Arch Otorhinolaryngol. 2018;22(2):152-156.

29. Wang Y, et al. Sublingual Immunotherapy Decreases Expression of Interleukin-33 in Children with Allergic Rhinitis. Indian J Pediatr. 2018;85(10):872-876.

30. Peng G, et al. Anti-IL-33 Antibody Has a Therapeutic Effect in an Atopic Dermatitis Murine Model Induced by 2, 4-Dinitrochlorobenzene. Inflammation. 2018;41(1):154-163.

31. Jang YH, et al. House Dust Mite Increases pro-Th2 Cytokines IL-25 and IL-33 via the Activation of TLR1/6 Signaling. J Invest Dermatol. 2017;137(11):2354-2361.

32. Londei M, Kenney B, Los G, Marino MH. A Phase 1 Study of ANB020, an anti-IL-33 monoclonal Antibody in Healthy Volunteers. J Allergy Clin Immunol. 2017;39(2_Suppl):AB73.

33. Kenny B, Los G, Marino MH, Londei M. A Phase 1 Study of ANB020, an Anti-IL-33 Monoclonal Antibody, in Healthy Volunteers. AnaptysBio. https://www2 anaptysbio.com/wp-content/uploads/ANB020-Phase-1-Study-Poster-AAD-andAAAAI-2017.pdf. Accessed October 18, 2019.

34. Chinthrajah RS, et al. Sustained Outcomes in a Large Double-1 blind, Placebo-controlled, Randomized Phase 2 Study of Peanut Immunotherapy. Lancet. 2019;394(10207):P1437-1449.

35. Komai-Koma M, et al. Interleukin-33 amplifies IgE synthesis and triggers mast cell degranulation via interleukin-4 in naïve mice. Allergy. 2012;67(9):1118-1126.

36. Kulis M, et al. High- and low-dose oral immunotherapy similarly suppress pro-allergic cytokines and basophil activation in young children. Clin Exp Allergy. 2019;49(2):180-189.

37. Ahmed A, Koma MK. Interleukin-33 Triggers B1 Cell Expansion and Its Release of Monocyte/Macrophage Chemoattractants and Growth Factors. Scand J Immunol. 2015;82(2):118-124.

38. Han H, Roan F, Johnston LK, Smith DE, Bryce PJ, Ziegler SF. IL-33 promotes gastrointestinal allergy in a TSLP-independent manner. Mucosal Immunol. 2018;11(2):394-403.

39. Hsu CL, Neilsen CV, Bryce PJ. IL-33 is produced by mast cells and regulates IgE-dependent inflammation. PLoS One. 2010;5(8):e11944.

40. Murakami-Satsutani N, et al. IL-33 Promotes the Induction and Maintenance of Th2 Immune Responses by Enhancing the Function of OX40 Ligand. Allergol Int. 2014;63(3):443-455.

41. Stier MT, et al. IL-33 Is a Cell-Intrinsic Regulator of Fitness during Early B Cell Development. J Immunol. 2019;203(6):1457-1467.

42. Sampson HA, et al. Standardizing double-blind, placebo-controlled oral food challenges: American Academy of Allergy, Asthma \& Immunology-European Academy of Allergy and Clinical Immunology PRACTALL consensus report. J Allergy Clin Immunol. 2012;130(6):1260-1274.

43. Syed A, et al. Peanut oral immunotherapy results in increased antigen-induced regulatory T-cell function and hypomethylation of forkhead box protein 3 (FOXP3). J Allergy Clin Immunol. 2014;133(2):500-510. 